Journal of Animal and Veterinary Advances 10 (15): 1994-2003, 2011

ISSN: $1680-5593$

(C) Medwell Journals, 2011

\title{
Fas-Associated Death Domain (FADD) Mediated Activation of a Apoptosis Program in Bovine Follicular Granulosa Cells
}

\author{
${ }^{1,2}$ Run-Jun Yang, ${ }^{2} \mathrm{Jun}-\mathrm{Ya} \mathrm{Li},{ }^{2}$ Xue Gao, ${ }^{1}$ Zhi-Hui Zhao, ${ }^{2}$ Lu-Pei Zhang, \\ ${ }^{2}$ Hui-Jiang Gao and ${ }^{2}$ Shang-Zhong $\mathrm{Xu}$ \\ ${ }^{1}$ College of Animal Science and Veterinary Medicine, \\ Jilin University, 130062 Changchun, P.R. China \\ ${ }^{2}$ Institute of Animal Sciences, Chinese Academy of Agricultural Sciences, \\ Yuanmingyuan West Road No. 2 Haidian District, 100193 Beijing, P.R. China
}

\begin{abstract}
Fas-Associated Death Domain (FADD) is a signal connection protein in the Fas/FasL system which may play a key role in apoptosis. Follicle atresia and luteolysis are thought to occur by apoptosis. To reveal the intracellular signal transduction molecules involved in the process of follicular development in the bovine ovary. This was accomplished by cloning the FADD gene using RT-PCR, deleting the termination codon in its cDNA and directionally cloning the amplified FADD gene into the eukaryotic expression vector pAcGFP-N1. The pAcGFP-bFADD recombinant plasmid was then transfected into bovine follicular granulosa cells by using lipofectamine 2000. Expression of AcGFP was observed under fluorescent microscopy and the transcription and expression of FADD was detected by RT-PCR and Western-blot. Then the Methyl-Tetrazolium (MTT) assay was performed to determine the growth inhibition of cells. Cell apoptosis was observed by using Hoechs 333342 staining and DNA-Ladder Method. The results showed that the pAcGFP-bFADD recombinant plasmid was successfully constructed. AcGFP expression was detected as early as $24 \mathrm{~h}$ after transfection. A $654 \mathrm{bp}$ fragment was amplified by RT-PCR and a $51.4 \mathrm{kD}$ target protein was detected by Western-blot. Cells viability decreased significantly at $72 \mathrm{~h}$ after transfection and the apoptosis rate of the cells transfected with pAcGFP-FADD was significantly higher than the control group. Cells in the FADD transfection group showed ladder patterns characteristic of apoptosis and the nuclei were shrunken and densely hyperchromatic or fragmented suggesting that FADD is capable of inhibiting the proliferation of bovine follicular granulosa cells and inducing cell apoptosis when over-expressed.
\end{abstract}

Key words: FADD, bovine follicle, apoptosis, granulosa cell, fragmented, China

\section{INTRODUCTION}

Apoptosis is an important process that maintains appropriate cell numbers by killing excess cells (Meier et al., 2000). The Fas/FasL (Fas Ligand) pathway is an important pathway of apoptosis that controls cell proliferation and tissue remodeling (Kersemaekers et al., 2002).

Morphological and biochemical studies have shown that the demise of both somatic and germ cells in the ovary is mediated by apoptosis (Fulton et al., 2005). Coordination between oocyte and granulosa cells is an essential prerequisite to normal follicular development (Shimizu et al., 2009). Studies in rat and bovine granulosa cells have demonstrated that cell-cell contact plays a vital role in inhibiting granulosa cell apoptosis and regulating proliferation (Cannon et al., 2005; Isobe and Yoshimura,
2007). Hence, a role for gap and tight junctions between granulosa cells and oocytes in preventing granulosa cell apoptosis has been proposed (Geng et al., 2008; Ikeda et al., 2006).

The Fas-Associated Death Domain (FADD) protein is an adapter/signaling molecule that has been shown to function in animal cells to promote apoptosis and to inhibit NF- $\kappa$ B activation (Chao et al., 2005). Subsequently, the researchers discovered that FADD plays a role not only in apoptosis signaling pathway but also in the proliferation of $\mathrm{T}$ cells; the gametogenesis and application of gene therapy also plays an important role (Bell et al., 2006; Luschen et al., 2005; Yu et al., 2009).

The bovine FADD gene is located on chromosome 29 and contains two exons which encode DED and DD protein domains, respectively. The FADD mRNA and protein are expressed extensively in the lymphoid tissue

Corresponding Author: Shang-Zhong Xu, Institute of Animal Sciences, Chinese Academy of Agricultural Sciences, 100193 Beijing, P.R. China 
and nonlymphoid tissues (such as liver, testis, pancreas, ovary and other tissues) and this trend is more significant in ovary, testis and lymphoid tissue (Szperka et al., 2005; Yamaguchi et al., 2007).

In different stages of oocyte development, FADD was involved in follicle atresia mediated apoptosis by Fas/FasL with interaction in different cytoplasmic proteins to maintain the equilibrium state of follicular development (Rangelova et al., 2008). It reveals that FADD may plays an important role in the regulation of oogenesis.

In the present study, we have inserted the cloned FADD gene into the eukaryotic expression vector pAcGFP-N1, successfully constructed fusion protein recombinant plasmid pAcGFP-bFADD and transfected it into bovine follicular granulosa cells. We were then able to detect the proliferation activities and apoptosis rate of cells transfected with the FADD gene. This technique can be used to provide basic research on regulation of FADD on the bovine oogonium development.

\section{MATERIALS AND METHODS}

Gene cloning and construction of mammalian cell expression vector for $\mathrm{pAcGFP-bFADD}$ fusion protein: Bovine ovaries were collected at a local abattoir and frozen rapidly in liquid nitrogen and then brought back to the laboratory. Total RNA was extracted from bovine ovary using Trizol kit (Intrivogen Corporation, Carlsbad, California, USA) and then reverse-transcribed using the cDNA synthesis reverse transcription kit (Takara, Dalian, China) to synthesize cDNA.

According to the FADD gene total length sequence (GenBank accession number: NM_001007816) a pair of primers was designed, forward 5'-CATGGACCCGTTCCT GGTGC-3' and reverse 5'-CACAGCCACCTCCC TGAGTC TTC-3'. PCR amplification cycles was performed as follows: $94^{\circ} \mathrm{C}$ for $90 \mathrm{sec} ; 35$ cycles of $94^{\circ} \mathrm{C}$ for $30 \mathrm{sec}, 65^{\circ} \mathrm{C}$ for $30 \mathrm{sec}$ and $72^{\circ} \mathrm{C}$ for $1 \mathrm{~min}$ and a final extension period at $72^{\circ} \mathrm{C}$ for $10 \mathrm{~min}$. The PCR products were purified and recovered using the agarose gel DNA recovery kit (Tiangen, Beijing, China). The purified FADD genes were ligated with pMD19-T vector (Takara, Dalian, China) and then were transformed into DH5 $\alpha$ competent cells. The positive clones were picked out and shaken overnight at $37^{\circ} \mathrm{C}$ and then a random analysis of 8 clones with PCR and sequencing analysis was conducted at Sinogenomax Company (Beijing, China).

The researchers designed primers at two ends of the FADD open reading frame and inserted $\operatorname{Bgl} \alpha$ restriction enzyme site in the upstream prime and four protective bases before ATG, A Kozak sequence was also included to increase the inserted gene expression level in eukaryotic cells. Forward primer was designed as follows: 5'-ACTAAGATCTGCCACCATGGACCCGTTCCTGGT-3' (AGATCT is the Bgl $\alpha$ enzyme site, GCCACCATG is the Kozak sequence). When the researchers designed the reverse primer, the stop codon TGA was deleted and the $\mathrm{C}$ base and EcoRI restriction enzyme site were inserted downstream of it. The FADD open reading frame should be aligned with the downstream $A C G F P$ gene sequence to ensure coexpression with the fusion protein. The reverse primer was designed as follows: 5'-ACTAGAATTCC GG AGGCTCCCGGGGCAGCG-3' (GAATTC is EcoRI enzyme site). The PCR product was recovered and cloned into pMD19-T simple vector and then it was transformed into DH5 $\alpha$ competent cells. The positive clones were picked out and shaken overnight at $37^{\circ} \mathrm{C}$. Plasmids were extracted from sense clones and digested with $\operatorname{Bgl} \alpha$ and EcoRI enzymes. A cDNA fragment of 654 bp was recovered and directly ligated to the pAcGFP-N1 eukaryotic expression vector that was digested with $\operatorname{Bgl} \alpha$ and EcoRI enzymes and transformed into $\mathrm{DH} 5 \alpha$ competent cells. The positive clones were picked out and shaken overnight at $37^{\circ} \mathrm{C}$.

Identification of recom binant plasmid pAcGFP-bFADD: After random analysis of 12 clones with PCR, plasmids were extracted from sense colonies and digested with Bgl $\alpha$ and EcoRI enzymes to confirm the expression of the bovine FADD. The DNA sequence of the ORF was determined using an automatic DNA sequencer (ABI Prism 310, Foster, CA, USA). All of these procedures were performed according to the manufacturer's instructions. The recombinant plasmid pAcGFP-bFADD was amplified in $\mathrm{DH} 5 \alpha$ cells and then the endotoxin free plasmid was extracted from the sense colonies using the EndoFree Plasmid Kit (Tiangen) and stored at $-20^{\circ} \mathrm{C}$.

G418 cytotoxicity test for bovine follicular granulosa cells: Bovine follicular granulosa cell lines were obtained from the Cell Center of Chinese Academy of Medical Sciences. The cells were plated on 24 well culture plates (Falcon, Franklin Lakes, NJ, USA) and incubated in a $\mathrm{CO}_{2}$ incubator (Thermo, Marietta, Ohio, USA) at $37^{\circ} \mathrm{C}$ for $24 \mathrm{~h}$, with $5 \% \mathrm{CO}_{2}$ in the air. After $24 \mathrm{~h}$ of culture, the DMEM medium (GIBCO, Iinvitrogen, Carlsbad, California, USA) supplemented with $10 \%(\mathrm{~V} / \mathrm{V})$ Fetal Bovine Serum (GIBCO) and 1\% (V/V) L-Glutamine (GIBCO) was replaced with DMEM medium containing different concentrations of G418 (100, 200, 300, 400, 500, 600, 700, 800, 900, $1000 \mu \mathrm{g} \mathrm{mL}^{-1}$; Sigma, St. Louis, MO, USA). Cells were incubated at $37^{\circ} \mathrm{C}$, in $5 \% \mathrm{CO}_{2}$ and the media was replaced every $72 \mathrm{~h}$ for 2 weeks of observation. The optimum concentration of G418 as a selection agent for follicular granulosa cell wasfound to be the lowest concentration 
under which all of the cells were killed 10-14 days after culture in DMEM with G418. We determined this concentration to be $600 \mu \mathrm{g} \mathrm{mL}$. After the cells spread out fully, positive clones were reselected using $600 \mathrm{~g} \mathrm{~mL}^{-1}$ of G418. Finally, cell clones which could stably express bovine $F A D D$ gene were chosen for subsequent analysis.

Transfection and fluorescence detection of fusion protein: About 1 day before transfection, $0.5-2 \times 10^{5}$ bovine follicular granulosa cells were plated in $500 \mu \mathrm{L}$ of growth medium without antibiotics per well of a 24 well culture plate (Falcon). When the cells reached $>90 \%$ confluency, the growth medium $[10 \%$ (V/V) Fetal Bovine Serum, $100 \mathrm{U} \mathrm{mL}^{-1}$ Penicillin-Streptomycin (GIBCO) and $1 \%$ (V/V) L-Glutamine] was replaced by Opti-MEM serum-free media (GIBCO). For transfection, DNA was diluted in $50 \mu \mathrm{L}$ Opti-MEM serum-free media and then mixed gently with Lipofectamine ${ }^{\mathrm{Tx}} 2000$ (GIBCO) before use and the appropriate amount was diluted in $50 \mu \mathrm{L}$ of Opti-MEM serum-free media and incubated for $5 \mathrm{~min}$ at room temperature. After $5 \mathrm{~min}$ of incubation, the diluted DNA was combined with diluted Lipofectamine ${ }^{\text {tu }} 2000$ (total volume $=100 \mu \mathrm{L}$ ) and was mixed gently and incubated for $20 \mathrm{~min}$ at room temperature. The $100 \mu$ DNA-Lipofectamine 2000 mixture was added to each well containing the cells and medium. The DNA-liposome complex transfers into cells and the anionic lipid of the membrane diffuses into the complex because the membrane loses its electrostatic balance. The anionic lipid of the membrane then combines with the positive ions of cationic liposomes, forming the neutral ion pair so that the pAcGFP-bFADD plasmid DNA break away from the DNA-liposome complex, enter the cytoplasm and then enter the nucleus through the nuclear pore. Finally, the bovine $F A D D$ gene encoding protein is produced by transcription and expression in the nucleus. The cells were incubated at $37^{\circ} \mathrm{C}$ in a $\mathrm{CO}_{2}$ incubator for $4-6 \mathrm{~h}$ and then the medium was changed to growth medium. The cells were put in a 1:10 or higher dilution of fresh growth medium $24 \mathrm{~h}$ after transfection. The positive cell clones were screened using G418. About $12 \mathrm{~h}$ later, the expression of AcGFP in the cells was observed under a fluorescence microscope (NikonTE2000, Japan) and the number of AcGFP-positive cells were counted under high power magnification every $24 \mathrm{~h}$.

Analysis of bovine FADD by RT-PCR and westernblotting: To confirm the insertion of a bovine FADD open reading frame, the bovine follicular granulosa cells were harvested after a stable transfection screening with G418. The mRNA was extracted from the cells using Quickprep
Micro mRNA Purification Kit (Invitrogen) and then reverse-transcribed to synthesize the cDNA. The primer for amplification of partial cDNA sequence of bovine FADD was designed as follows: forward 5'-ACTA AGATCTGCCACCATGGACCCGTTCCTGGT-3' and reverse 5'-ACTAGAATTCCGGAGGCTGGGCAGCG-3'.

The other cells were washed twice with PhosphateBuffered Saline (PBS, pH 7.4), treated with $10 \%$ (V/V) trichloro acid (Wako Pure Chemical Industries, Osaka, Japan) at $4^{\circ} \mathrm{C}$ for $30 \mathrm{~min}$ and scraped off. These cells were then suspended in UTD buffer [ $9 \mathrm{~mol} \mathrm{~L}^{-1}$ Urea (Wako), $2 \%(\mathrm{~V} / \mathrm{V})$ Triton X-100 (Sigma) and 1\% (W/V) $( \pm)-$ Dithiothreitol (Wako)] and 2\% (W/V) lithium dodecyl sulfate (Wako).

The whole cell lysate was separated by $15 \%$ (W/V) gradient Sodium Dodecylsulfate-Polyacrylamide Gel Electrophoresis (SDS-PAGE) and then transferred onto Polyvinylidene Difluoride (PVDF) membranes (Bio-Red laboratories Inc, USA). The PVDF membranes were stained with a $0.2 \%$ (W/V) Ponceau-S solution (Sigma) at $25^{\circ} \mathrm{C}$ for $1 \mathrm{~min}$ and then immersed in blocking solution [20 mM Tris- $\mathrm{HCl}$ (pH 7.6), $137 \mathrm{mM} \mathrm{NaCl}$ and $0.1 \%$ (V/V) Tween-20 containing 5\% (W/V) skim milk (Sigma)] for $30 \mathrm{~min}$. They were then incubated with rabbit anti-bovine FADD polyclonal antibody (Santa Cruz Biotechnology Inc, Santa Cruz, CA,USA) at $4^{\circ} \mathrm{C}$ for $12 \mathrm{~h}$. After a wash with blocking solution, they were incubated with Horseradish Peroxidase (HRP)-conjugated goat anti-rabbit IgG antibody (Golden Bridge, Beijing, China) at $25^{\circ} \mathrm{C}$ for $1 \mathrm{~h}$. Chemiluminescence was visualized using an ECL system (Applygen Technologies Inc, Beijing, China) according to the manufacturer's direction.

Detection of the proliferation activities of transfected cells by MTT assay: Bovine follicular granulosa cells (the cell line was obtained from the Cell Center of Chinese Academy of Medical Sciences) that were in exponential growth phases were collected, the cell density was adjusted to $1 \times 10^{6} \mathrm{~mL}^{-1}$ and the cells were added to 96 well culture plates with $100 \mu \mathrm{L}$ per well. Cells were cultured for $12 \mathrm{~h}$ at $37^{\circ} \mathrm{C}$ in an incubator containing $5 \%$ $\mathrm{CO}_{2}$ and then abandoned the medium. Comparisons were made between three groups: a blank control group, consisting of $50 \mu \mathrm{L}$ per well serum free DMEM; the experimental group, consisting of $50 \mu \mathrm{L}$ per well of the pAcGFP-bFADD plasmid and Lipofectami ${ }^{\text {TM }} 2000$ mix and the negative control, consisting of $50 \mu \mathrm{L}$ per well of the pAcGFP-N1 plasmid and Lipofectami ${ }^{\mathrm{TM}} 2000$ mix. All groups were cultured for $4 \mathrm{~h}$ at $37^{\circ} \mathrm{C}$ in a $\mathrm{CO}_{2}$ incubator containing $5 \% \mathrm{CO}_{2}$, after which the medium was discarded. Then, $200 \mu \mathrm{L}$ DMEM (containing about $10 \%$ of the womb bovine serum) was added to each well and then 
$10 \mu \mathrm{L}$ MTT ( $5 \mathrm{mg} \mathrm{mL}^{-1}$, Sigma,St. Louis, MO, USA) was subsequently added at $0,24,48,72,96$ or $120 \mathrm{~h}$ and the cells continued to culture for $4 \mathrm{~h}$. The media was then exchanged for $200 \mu \mathrm{L}$ DMSO (GIBCO, Iinvitrogen, Carlsbad, California, USA) per well and the plate was oscillated at a low speed for $10 \mathrm{~min}$. OAfter intensive dissolving with Formazan, the $\mathrm{OD}_{570}$ value was detected with Micro plate spectrophotometer. The cells' growth curve was obtained using the value of $O D_{570}$ as the ordinate and time (h) as the abscissa and the cell growth inhibition rate was calculated.

\section{Detection of apoptosis of transfected cells using Hoechst} 33342 staining and DNA Ladder Methods: About $48 \mathrm{~h}$ after transfection, bovine follicular granulosa cells were centrifuged and the supernatant was removed after trypsin digestion. The cells were washed twice with PBS, Hoechst 33342 (Sigma) was added and the cells were cultured for $15 \mathrm{~min}$ at $37^{\circ} \mathrm{C}$ in the dark. Cells were examined using fluorescence microscopy. Cells were randomly selected for examination using high power magnification ( $\mathrm{x} 400$ ), photographed and both normal cells (marked by big nuclear, dispersion and homogeneous fluorescence) and the apoptotic cells (indicated by nuclear shrinkage and hyperchromatic nuclei) were counted. About 12 samples were taken in from each group, counted and 100 cells were counted for each sample. The apoptosis rate was calculated according to the following formula:

$$
\text { Apoptosis rate }=\frac{\text { Apoptosis cells }}{(\text { Normal cells }+ \text { Apoptotic cells })} \times 100 \%
$$

The bovine follicular granulosa cells were collected at $72 \mathrm{~h}$ after transfection and the cell density was adjusted to $1 \times 10^{7} \mathrm{~mL}^{-1}$, according to the DNA Ladder kit's instruction (GIBCO, Iinvitrogen, Carlsbad, California, USA). The DNA was extracted, dissolved and precipitated with $17 \mu \mathrm{L}$ double distilled water and $3 \mu \mathrm{L} 6 \times$ Loading
Buffer was added and well-mixed. The entire $20 \mu \mathrm{L}$ DNA solution was added to a $1.0 \%$ agarose gel for electrophoresis and resulting fragments were observed with UV transilluminator.

Statistical analysis: Experimental data were shown as Mean+/-SD. Statistically significant differences in the mean were determined using t-tests. Statistically significant differences were defined as $\mathrm{p}<0.05$.

\section{RESULTS AND DISCUSSION}

\section{Construction and identification of eukaryotic expressing} vector of fusion gene bFADD-AcGFP: The 654 bp encoding region of the FADD gene was amplified from the pT-bFADD plasmid with specific primers by Touchdown PCR (TD-PCR). The expected fragments were obtained by complete digestion of the PMD19-T-FADD plasmid which was extracted from the transformed positive clones and digested using Bgl $\alpha$ and EcoRI. The target gene fragment was successfully connected to the $5^{\prime}$ end of the AcGFP cDNA which was confirmed to have the FADD reading frame aligned with AcGFP. The $654 \mathrm{bp}$ fragments were obtained by complete digestion of the recombinant plasmid pAcGFP-bFADD which was extracted from the transformed positive clones using Bgl $\alpha$ and EcoRI (Fig. 1a). The plasmids were extracted from positive clones and digested with Bgl $\alpha$ and EcoRI enzyme for $6 \mathrm{~h}$ at $37^{\circ} \mathrm{C}$ following the supplier's direction. M: DNA marker DL 5000; 1:pAcGFP-bFADD recombinant plasmid; 2,3: pAcGFP-bFADD digestion by restrictive enzyme $\mathrm{Bgl} \alpha$ and EcoRI.

The sequence analysis showed that the bovine FADD gene was successfully cloned into $\mathrm{Bg} 1 \alpha / \mathrm{EcoRI}$ site of the pAcGFP-N1 vector. The researchers confirmed that the FADD coding region sequence and the AcGFP gene sequence had the same reading frame. This was achieved through deleting the stop codon TGA and inserting the $\mathrm{C}$ base (Fig. 1b) such that the target gene and fusion protein gene could be expressed at the same time. The

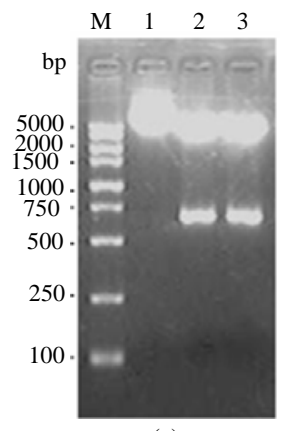

(a)

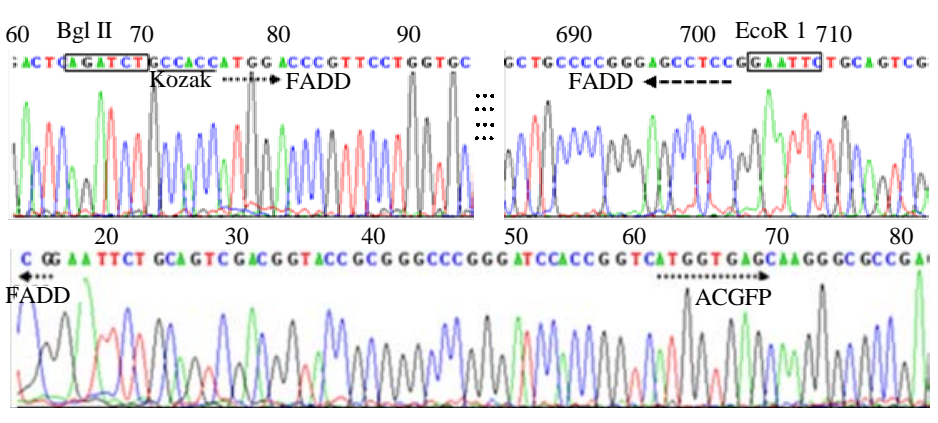

(b)

Fig. 1: Identification of recombinant plasmid pAcGFP-bFADD by restriction enzyme digestion and sequence analysis 
Table 1: Cytotoxicity test of G418 to cultured follicular granulosa cells for 12 days

G418 concentration

\begin{tabular}{lllllllllll}
$\left(\mu \mathrm{g} \mathrm{mL} \mathrm{mL}^{-1}\right)$ & 100 & 200 & 300 & 400 & 500 & 600 & 700 & 800 & 900 & 1000 \\
\hline
\end{tabular}

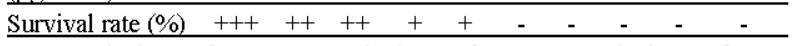

++ : survival rate of $80 \%$; ++: survival rate of $50 \%$; + survival rate of $30 \%$;

-: survival rate of $0 \%$

pAcGFP-bFADD plasmids were extracted from positive clones and sequenced by SinoGeneMax Company Limited. A: FADD ORF sequence of pAcGFP-bFADD, digestion sites with $\operatorname{Bgl} \alpha$ and EcoRI, Kozak sequence (digestion sites are in the box, underline part is Kozak Sequence, dotted line arrow direction is FADD ORF); B: AcGFP sequence of pAcGFP-bFADD recombinant (dotted line arrow direction on the right). The reconstructed plasmid was named the pAcGFP-bFADD vector.

Determination of the minimum dose of G418 for follicular granulosa cells: After 3 days selection with different concentrations of G418, the bovine follicular granulosa cells were found to be in differing degrees of death with the number of suspended and broken cells increasing in treatments supplemented with higher than $600 \mu \mathrm{g} \mathrm{mL}^{-1} \mathrm{G} 418$. Peak mortality was in the 8th-10th day exposure duration and the cells treated with $600 \mu \mathrm{g} \mathrm{mL}$ G418 or more were dead by the 10th day. The concentration of $600 \mu \mathrm{g} \mathrm{mL} \mathrm{mL}^{-1}$ was considered as the minimum dose of G418 for follicular granulosa cells to cause cell death (Table 1).

Transfection of follicular granulosa cells with pAcGFPbFADD plasmid and G418 selection of resistant cell strains: The positive charge of the cationic liposome's surface and the phosphate backbone of pAcGFP-bFADD plasmid DNA stably combine by electrostatic interaction to form the DNA-liposome complex. The complex is adsorbed to the cell membrane with the negative charge and then the DNA complex transfers into the cells and forms the inclusion bodies in the cytoplasm by fusion, osmosis of cytomembrane and endocytosis.

Cells transfected with the pAcGFP-bFADD plasmid by Lipofectamine 2000 were screened with G418 up to the 14 th day. The negative control cells were all dead but cell clones formed in experimental conditions. Subsequently the maintaining dose of $\mathrm{G} 418$ ( $600 \mathrm{ug} \mathrm{mL}^{-1}$ ) was used to the 18 th day when all cell degeneration and necrosis disappeared and the resistant cells formed positive clones and gradually proliferated. The expression of AcGFP was detected in the plasma and nucleus using fluorescent microscopy.

Evaluation of expression product by RT-PCR and Western blot analysis: The observation result of green fluorescence in the cells showed that the untransfected cells were not observed under microscope fluorescent and AcGFP could be observed around the lateral region of the nucleus in the follicular granulosa cells transfected with pAcGFP-bFADD and uniform distribution throughout the whole cell in the pAcGFP-N1 transfection group (Fig. 2a).

The expression of AcGFP-bFADD fusion protein and AcGFP in follicular granulosa cells after transfection. After transfection, the fluorescence could be detected in follicular granulosa cells transfected with either pAcGFP-bFADD or pAcGFP-N1 plasmid while no AcGFP expression was detected in untransfected follicular granulosa cells. AcGFP could be observed in the nucleus and its lateral region in pAcGFP-bFADD transfected cells while there was uniform distribution throughout the whole cell in pAcGFP-N1 transfected cells. A, B, C: transfected follicular granulosa cells under fluorescent microscope; D, E, F: transfected follicular granulosa cells under visible light. A, D: control group; B,E: pAcGFPbFADD transfection group; $\mathrm{C}, \mathrm{F}$ : pAcGFP-N1 transfection group.

The RNA of the monoclonal cells screened by G418 was extracted. A bright $654 \mathrm{bp}$ fragment was amplified in the pAcGFP-bFADD transfected follicular granulosa cells by RT-PCR whereas the 654 bp strap was weak in the pAcGFP-N1 transfected cells and the negative control cells (Fig. 2b). Total RNA was extracted from follicular granulosa cells and cDNA was prepared using universal primer. Specificity primers were used to amplify the FADD sequence, a $654 \mathrm{bp}$ fragment was detected by electrophoresis on $1.2 \%$ agarose gel in pAcGFP-bFADD transfection group. M: DNA marker DL 5000; 1: Control group; 2: pAcGFP-N1 transfection group; 3, 4: pAcGFPbFADD transfection group. The results show effective expression of FADD in the pAcGFP-bFADD transfected follicular granulosa cells, suggesting that the pAcGFPbFADD successfully transfected the follicular granulosa cell.

SDS-PAGE analysis indicated that the fusion protein of bFADD-AcGFP was expressed in pAcGFP-bFADD transfected cells and its molecular weight was about $51.4 \mathrm{kD}$ (Fig. 2c, lane 3, 4). Protein sample were loaded onto $12 \%$ SDS-PAGE to separate protein and transferred to nylon cellulose membrane. The membrane was probed with anti-FADD polyclonal antibody and then was probed with peroxidase-conjugated goat anti-rabbit polyclonal antibody as the second antibody. Bound antibodies were detected with the ECL (c1:M, protein molecular weight marker (MW marker); 1, cell lysate of cell of control group; 2, cell lysate of cell of pAcGFP-N1 transfection group; 3 , 4 , cell lysate of cell of pAcGFP -bFADD transfection group; c2: 1, Western blotting analysis of pAcGFP-N1 transfection group; 2, Western blotting analysis of 

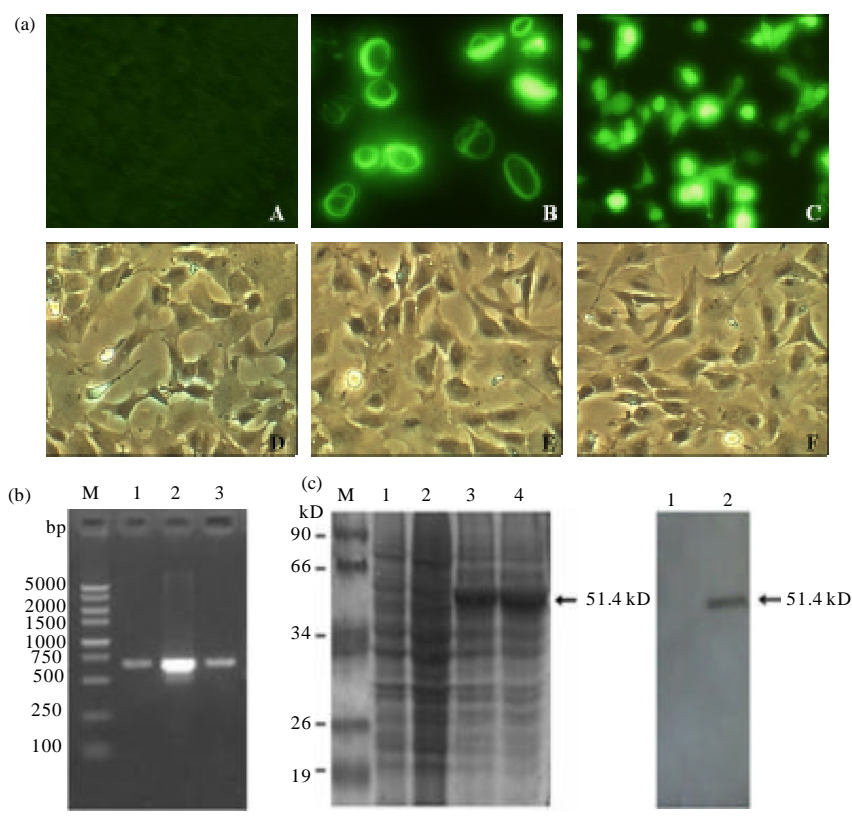

Fig. 2: The expression of bovine FADD on follicular granulosa cells determined by RT-PCR and Western blotting

pAcGFP-bFADD transfection group). No expression of the fusion protein of bFADD-AcGFP was detected in pAcGFP-N1 transfected cells or negative control cells (Fig. 2c, lane 1,2). These results serve as preliminarily evidence that follicular granulosa cells transfected with AcGFP expression vectors of the bovine $F A D D$ gene are capable of expressing fusion target proteins. The expressed fusion protein showed specificities of FADD polyclonal antibody as proved by Western blot and further proved to be an immunoreactive protein (Fig. 2c). FADD is known to induce apoptosis of ovarian granulosa cells, leading to follicular atresia so it is possible that it helps maintain the equilibrium state of bovine follicular development.

Detection of the proliferation activities of follicular granulosa cells transfected with FADD using the MTT assay: The growth curve of bovine follicular granulosa cells was assessed using the MTT method 0-5 days after transfection with the pAcGFP-bFADD and pAcGFP-N1 plasmids. The growth curve of pAcGFP-N1 treatment had no significant difference compared to non-transfected controls but the growth curve of pAcGFP-FADD transfected cells was significantly lower than controls (Fig. 3a). The growth curves of bovine follicular granulosa cells. These results indicate that the pAcGFP-bFADD plasmid inhibited the growth of granulosa cells, especially $72 \mathrm{~h}$ after transfection. The growth of about $87.6 \%$ bovine follicular granulosa cells was inhibited after 5 days following pAcGFP-bFADD plasmid transfection and there was a significant positive correlation between rate
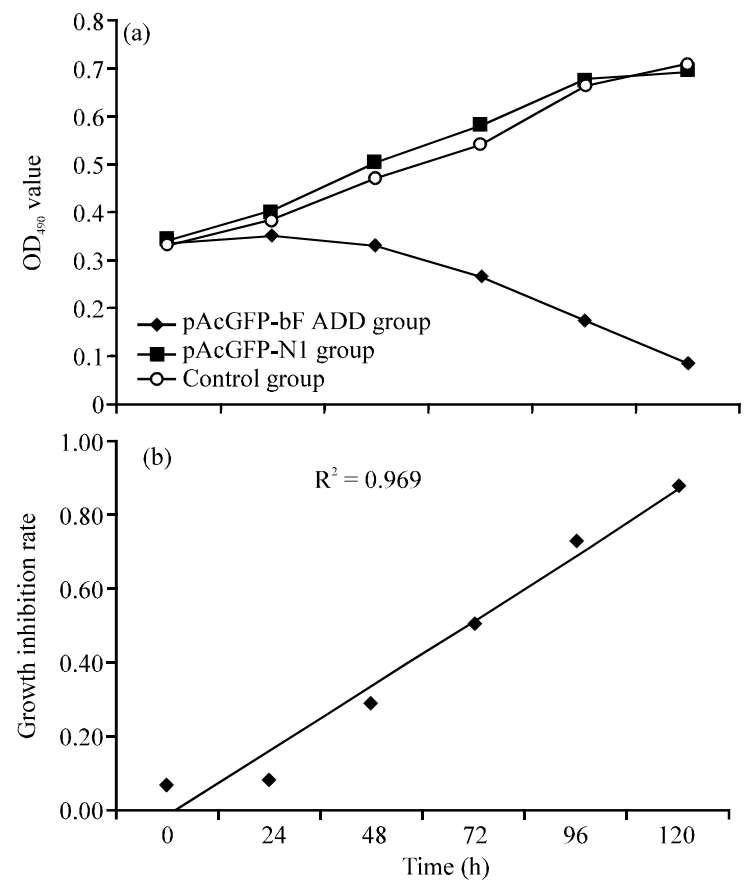

Fig. 3: Proliferation activities detected in bovine follicular granulosa cells. The growth curve of cattle granulosa cells was determined using the MTT method following transfection with pAcGFPFADD or pAcGFP-N1 plasmids for $0 \sim 5$ days

of growth inhibition and transfection time (Fig. 3b). The growth inhibition rates of the bovine follicular granulosa cells. 


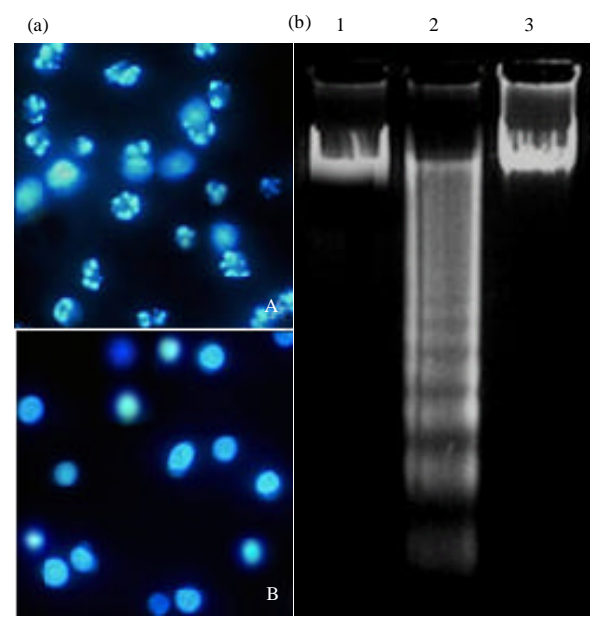

Fig. 4: Detection of apoptosis in transfected follicular granulosa cells

The effect of FADD over-expression in follicular granulosa cells: After staining with Hoechst 33342, the normal bovine follicular granulosa cells showed large nuclei with dispersed fluorescence while the apoptotic granulosa cell nuclei showed chromatin fragmentation with pyknotic fluorescence (Fig. 4a). Fluorescent micrographs of bovine follicular granulosa cells stained with Hoechst 33342. After staining with Hoechst 33342 , normal bovine follicular granulosa cells had larger nuclei with dispersed fluorescence (B) while apoptotic granulosa cells nuclei showed chromatin fragmentation with pyknotic fluorescence (A). The average apoptosis rate of follicular granulosa cells was significantly higher in cells transfected with the pAcGFP-FADD plasmid (31.28 $3.47 \%$ ) compared to cells transfected with the pAcGFP-N1 plasmid $(5.46 \pm 2.86 \%)(\mathrm{p}<0.01)$.

The genomic DNA was extracted after transfection with pAcGFP-bFADD or pAcGFP-N1 plasmids and analyzed by agarose gel electrophoresis. Results show that genomic DNA of granulosa cells transfected with the pAcGFP-bFADD plasmid was dispersed with bands which were more visible with the increasing time of treatment while the genomic DNA of follicular granulosa cell transfected with pAcGFP-N1 plasmid appeared to be normal (Fig. 4b). Detection of apoptosis of transfected cells using the DNA Ladder Method. The genomic DNA was extracted after transfection with pAcGFP-bFADD or pAcGFP-N1 plasmids at $72 \mathrm{~h}$ post-transfection and analyzed by agarose gel electrophoresis. The genome DNA of granulosa cells transfected with the pAcGFP-bFADD plasmid showed many dispersed bands which became more visible with treatment exposure time whereas the control group showed normal bands.
(1, Genomic DNA of control group granulosa cells; 2 , Genomic DNA of granulosa cells transfected with pAcGFP-bFADD plasmid; 3. Genomic DNA of granulosa cells transfected with pAcGFP-N1 plasmid.

Apoptosis is an important phenomenon involved in cell survival and death during differentiation and development. The death ligand and receptor systems are consi dered to be apoptosis-inducing factors (Yamamoto et al., 2003).

Apoptosis can be mediated by caspase- 8 activation via the extrinsic or death receptor-mediated pathway, resulting in formation of the Death-Inducing Signaling Complex (DISC) containing the adapter molecule FADD and procaspase-8 (Carrington et al., 2006; Inoue et al., 2007).

An adaptor protein such as the Fas-Associating Death Domain protein (FADD) which has a DD in the Cterminal region and a Death Effector Domain (DED) in the $\mathrm{N}$-terminal region is recruited when each specific ligand binds to its receptor. FADD, in turn can recruit procaspase-8 via protein-protein interactions of Death Effector Domains (DED) contained within both proteins. FADD recruitment of the procaspase- 8 molecules enables activation of these pro-enzymes into fully functional proteases resulting in the onset of apoptosis. FADD binds with procaspase- 8 which composes the DeathInducing Signaling Complex (DISC). As a result of the proteolytic autoactivation of procaspase- 8 , subunit protein $18(\mathrm{pl})$ and $11(\mathrm{p} 11)$ are released into the cytoplasm and reform a proteolytic component that cleaves many substrate proteins such as downstream caspase, procaspase- 3 and mitochondrion stimulator Bid. Thus, FADD is a key molecule in the intracellular apoptosis signal-inducing system (Balachandran et al., 2004; Scott et al., 2009).

A previous study about an analyzing expression map in the laboratory suggested mRNA of the bovine FADD, highly expressed in lymphoid tissue, ovary and testis whereas less expressed in other tissues. This indicated that FADD in the lymphoid tissue played an important role in keeping the bovine immune environment stable. The Fas in testicular germ cells and ovary oocytes and FasL in sertoli cells and follicular granulosa cells interacted which could keep the spermatogenesis and oogenesis balanced, through regulating the FADD expression level. Gene mutation or abnormal expression of FADD in the reproductive system, leading to internal environment disorder and abnormal spermatogenesis and oogenesis could cause bull's dyszoospermia or aspermia and reduce a cow's ovulation rate and conception rate (Fischer et al., 2006; Khatib et al., 2009). 
When the researchers constructed the eukaryotic expression vector for the pAcGFP-bFADD fusion protein, the researchers took advantage of directional cloning by introducing Bgl $\alpha$ (AGATCT) and EcoRI (GAATTC) at two sites in the upstream primer and downstream primer, respectively. These two restriction enzymes produced different 3 'cohesive ends which allowed the target gene to be directionally connected to vector. The benefits of this method are as follows: the vector fragment could not be cyclized since the vector's two cohesive ends did not complement each other so, there were few false positive recombinant clones because the foreign bovine FADD gene was inserted into recombinant plasmid in one direction, it was not necessary to screen for the right connection and restriction enzyme sites were preserved which was beneficial for further identification.

Kozak analyzed the relationship between sequence of the mRNA $5^{\prime}$ end and the translation efficiency in the eucaryotic expression gene and found that a $5^{\prime} \mathrm{G} / \mathrm{N}-\mathrm{C} / \mathrm{N}-$ C/N-ANNATGG 3' sequence could improve transcription and translation efficiency (Kozak, 1980). Notably, positioning $A$ in the -3 site and $G$ in the +4 site was important for improving the translation efficiency. Therefore, here the Kozak sequence was introduced after the upstream primer's Bgl $\alpha$ site to promote high expression of the $F A D D$ gene in the recombinant plasmid.

G418 is one of the aminoglycoside antibiotics which is toxic to both prokaryotic cells and eukaryotic cells. It is usually utilized to screen transfections via insertion of a neo gene into the genome of eukaryotic cells (Yallop and Svendsen, 2001). Once the sequence coded by the neo gene starts to transcribe into mRNA, the amino glycoside phosphotransferase which is toxic to the cells becomes highly expressed. As such cells were grown up in a selective medium that included G418. It should be noted that it is important to test for the optimal G418 screening concentration since there may be varying sensitivities of different cells to G418 as well as varying concentrationdependent activities of $\mathrm{G} 418$ depending on the vendor source. Under the conditions of these experiments, all the cells died when treated with a concentration of $600 \mu \mathrm{g} \mathrm{mL}^{-1} \mathrm{G} 418$ for 12 days so the researchers chose the $600 \mu \mathrm{g} \mathrm{mL}^{-1}$ as the best screening concentration. During the screening, the researchers initially selected $600 \mu \mathrm{g} \mathrm{mL}^{-1}$ of $\mathrm{G} 418$ when clones appeared. However, the researchers then selected $200 \mu \mathrm{g} \mathrm{mL}^{-1}$ of G418, instead of $600 \mu \mathrm{g} \mathrm{mL} \mathrm{m}^{-1}$ so that the cells grew up rapidly.

pAcGFP-bFADD was transfected into follicular granulosa cell mediated by Lipofectami 2000 with a transfection efficiency reaching $76 \%$. After screening for 2 weeks using $600 \mu \mathrm{g} \mathrm{mL}^{-1}$ of $\mathrm{G} 418$, the positive clones emited fluorescence indicating that the bovine FADD gene was completely inserted into the follicular granulosa cell genome and the fusion protein was stably expressed.
Green fluorescence protein (AcGFP-bFADD) was observed in the cell cytoplasm around the nucleus, in the shape of a loop, under a high power fluoroscope. It was close to the report that was made by other researchers who observed that FADD lay in the cell cytoplasm. As a signal protein, it connected the death receptor and cytoplasm which could transfer extracellular apoptosis signal into the cytoplasm to activate enzyme-related apoptosis and induce apoptosis. But because the researchers took the widely expressed CMV as AcGFP$\mathrm{N} 1$ 's promoter, they were not sure FADD was localized in the cytoplasm, it needed to be studied further.

The molecular weight of the green fluorescent protein was $28 \mathrm{kD}$ and the bovine FADD's molecular weight was $23.4 \mathrm{kD}$ so the fusion protein's molecular weight was about $51.4 \mathrm{kD}$, consistent with the detection using SDS-PAGE electrophoresis and Western blotting. Furthermore, FADD's antibody binding to the NC membrane showed a specific reaction with the fusion protein indicating that the follicular granulosa cells transfected with pAcGFP-bFADD highly expressed the immunocompetent FADD protein. Additional variables such as decreasing the concentration of coloured solution, increasing the rinse time, increasing the buffer volume and shortening exposure time could improve the protein immunoblotting ECL development effect.

The viability of granulosa cells was measured using the MTT method and the results showed that the growth curve of granulosa cells transfected with pAcGFP-N1 had no significant difference compared to non-transfected control cells but the growth curve of granulosa cells transfected with pAcGFP-bFADD was significantly lower compared to controls. This phenomenon was more pronounced after $72 \mathrm{~h}$ treatment and the growth was inhibited in $87.6 \%$ of the granulosa cells by 5 days posttransfection. These results suggest that the expression of FADD in granulosa cells could induce the apoptosis.

Hoechst 33342 is a fluorescence dye with a high membrane permeability that enters normal cells and binds with genomic DNA without injuring the cells. Both normal, viable cells and apoptotic cells can be dyed by Hoechst 33342 (Tatischeff et al., 2008). The nuclei of normal/viable cells appears as a light blue circle with deep blue granulations while the nuclei of apoptotic cells appears shrunken and fragmented due to contraction of the nucleus (Kaminsky et al., 2007). The fluorescence intensity of Hoechst 33342 was stronger in apoptotic cells than normal cells. The degree of apoptosis by FADD expression was tested using Hoechst 33342 dyeing and the results showed that transfection with the pAcGFPbFADD plasmid induces significant apoptosis in cattle ovary granulosa cells. One study reported the presence of many apoptotic granulosa cells in atretic follicles using cell ultrastructural analysis, a phenomenon which rarely, if ever, happens in healthy ovarian follicles (Yu et al., 
2004). The $\mathrm{Ca}^{2+} / \mathrm{Mg}^{2+}$ dependent nucleate endonuclease was shown to be activated in the cytoplasm during apoptosis and the genomic DNA was cut between nucleosomes to form $180-200$ bp DNA fragments (Ogata et al., 2003). The DNA fragments appeared as specific bands in agarose gel electrophoresis, a phenomenon that is a key marker for apoptosis. In the current study, the genomic DNA of cattle granulosa cells transfected with the pAcGFP-bFADD plasmid was analyzed using agarose gel electrophoresis which revealed the appearance of these specific bands. This phenomenon was similar to analogous studies in rat, sheep and cattle follicular granulosa cells (Choi et al., 2004; Han et al., 2006; Khandoker et al., 2001), confirming the effect of apoptosis in granulosa cells by FADD expression.

\section{CONCLUSION}

The study concludes that by fusing the bovine $F A D D$ gene to the AcGFP gene, the mammalian expression vector of the pAcGFP-bFADD fusion protein was constructed and found that transfection with the pAcGFP-bFADD plasmid induces significant apoptosis in cattle ovary granulosa cells. Suggesting that FADD is capable of inhibiting the proliferation of bovine follicular granulosa cells and inducing cell apoptosis when over-expressed. This result could provide technical support for basic research on the regulation of FADD on bovine oogonium development and become important for further research in the field of bovine development and reproduction.

\section{ACKNOWLEDGEMENTS}

This research was supported by National Natural Science Foundation of China (No.31000991) and the National R. and D. Project of Transgenic Organisms of Ministry of Science and Technology of China (No. 2009ZX08007-005B and No. 2009ZX08009-156B). The researchers would like to thank Ms. Yumei Yang for assistance with experimental protocols. The researchers are grateful to Dr. Xiaohui Zhang for cell culture technical assistance.

\section{REFERENCES}

Balachandran, S., E. Thomas and G.N. Barber, 2004. A FADD-dependent innate immune mechanism in mammalian cells. Nature, 432: 401-405.

Bell, B.D., A.F. Arechiga and C.M. Walsh, 2006. The role of Fas-associated death domain and caspase 8 in lymphocyte proliferation and survival. J. Immunol., 176: S206-S206.
Cannon, J.D., M. Cherian-Shaw and C.L. Chaffin, 2005. Proliferation of rat granulosa cells during the periovulatory interval. Endocrinology, 146: 414-422.

Carrington, P.E., C. Sandu, Y.F. Wei, J.M. Hill and G. Morisawa et al., 2006. The structure of FADD and its mode of interaction with procaspase-8. Mol. Cell, 22: 599-610.

Chao, W., Y. Shen, L. Li, H. Zhao, S.E. Meiler, S.A. Cook and A. Rosenzweig, 2005. Fas-associated death-domain protein inhibits TNF-alpha mediated NF-kappaB activation in cardiomyocytes. Am. J. Physiol. Heart Circ. Physiol., 289: H2073-H2080.

Choi, D., S. Hwang, E. Lee, S. Yoon, B.K. Yoon and D. Bae, 2004. Expression of mitochondria-dependent apoptosis genes (p53, Bax and Bcl-2) in rat granulosa cells during follicular development. J. Soc. Gynecol. Invest., 11: 311-317.

Fischer, C., M. Drillich, C. Gabler, W. Heuwieser and R. Einspanier, 2006. Postpartum reproductive failure in cattle: Is the examination of the gene expression in the bovine endometrium a way to success. Berl. Munch. Tierarztl. Wochenschrift, 119: 197-202.

Fulton, N., S.J.M. da Silva, R.A.L. Bayne and R.A. Anderson, 2005. Germ cell proliferation and apoptosis in the developing human ovary. J. Clin. Endocrinol. Metab., 90: 4664-4670.

Geng, L.Y., M. Fang, F. Jiang, M.D. Muhammad, L.G. Yang and Q.Y. Wen, 2008. Role of overexpression of inhibin $\alpha(1-32)$ fragment in bovine granulosa cell proliferation, apoptosis, steroidogenesis and development of co-cultured oocytes. Reprod. Domestic Anim., 43: 205-206.

Han, Z.B., G.C. Lan, Y.G. Wu, D. Han, W.G. Feng, J.Z. Wang and J.H. Tan, 2006. Interactive effects of granulosa cell apoptosis, follicle size, cumulusoocyte complex morphology and cumulus expansion on the developmental competence of goat oocytes: A study using the well-in-drop culture system. Reproduction, 132: 749-758.

Ikeda, S., K. Saeki, H. Imai and M. Yamada, 2006. Abilities of cumulus and granulosa cells to enhance the developmental competence of bovine oocytes during in vitro maturation period are promoted by midkine: A possible implication of its apoptosis suppressing effects. Reproduction, 132: 549-557.

Inoue, N., F. Matsuda-Minehata, Y. Goto, K. Sakamaki and N. Manabe, 2007. Molecular characteristics of porcine Fas-Associated Death Domain (FADD) and procaspase-8. J. Reprod. Dev., 53: 427-436.

Isobe, N. and Y. Yoshimura, 2007. Deficient proliferation and apoptosis in the granulosa and theca interna cells of the bovine cystic follicle. J. Reprod. Dev., 53: 1119-1124. 
Kaminsky, Y.G., E.A. Kosenko, N.I. Venediktova, V. Felipo and C. Montoliu, 2007. Apoptotic markers in the mitochondria, cytosol and nuclei of brain cells during ammonia toxicity. Neurochem. J., 1: 78-85.

Kersemaekers, A.M.F., P.C. van Weeren, J.W. Oosterhuis and L.H.J. Looijenga, 2002. Involvement of the Fas/FasL pathway in the pathogenesis of germ cell tumours of the adult testis. J. Pathol., 196: 423-429.

Khandoker, M.A., K. Imai, T. Takahashi and K. Hashizume, 2001. Role of gelatinase on follicular atresia in the bovine ovary. Biol. Reprod., 65: 726-732.

Khatib, H., W. Huang, X. Wang, A.H. Tran and A.B. Bindrim et al., 2009. Single gene and gene interaction effects on fertilization and embryonic survival rates in cattle. J. Dairy Sci., 92: 2238-2247.

Kozak, M., 1980. Binding of wheat germ ribosomes to bisulfite-modified reovirus messenger RNA: Evidence for a scanning mechanism. J. Mol. Biol., 144: 291-304.

Luschen, S., M. Falk, G. Scherer, S. Ussat, M. Paulsen and S. Adam-Klages, 2005. The Fas-associated death domain protein/caspase-8/c-FLIP signaling pathway is involved in TNF-induced activation of ERK. Exp. Cell Res., 310: 33-42.

Meier, P., A. Finch and G. Evan, 2000. Apoptosis in development. Nature, 407: 796-801.

Ogata, M., O. Inanami, M. Nakajima, T. Nakajima, W. Hiraoka and M. Kuwabara, 2003. $\mathrm{Ca}^{2+}$-dependent and caspase-3-independent apoptosis caused by damage in golgi apparatus due to $2,4,5,7$-tetrabromorhodamine 123 bromide-induced photodynamic effects. Photochem. Photobiol., 78: 241-247.

Rangelova, S., S. Kirschnek, A. Strasser and G. Hacker, 2008. FADD and the NF-kappa B family member Bcl-3 regulate complementary pathways to control T-cell survival and proliferation. Immunology, 125: 549-557.

Scott, F.L., B. Stec, C. Pop, M.K. Dobaczewska and J.J. Lee et al., 2009. The Fas-FADD death domain complex structure unravels signalling by receptor clustering. Nature, 457: 1019-1022.
Shimizu, T., N. Kosaka, C. Murayama, M. Tetsuka and A. Miyamoto, 2009. Apelin and APJ receptor expression in granulosa and theca cells during different stages of follicular development in the bovine ovary: Involvement of apoptosis and hormonal regulation. Anim. Reprod. Sci., 116: 28-37.

Szperka, M.E., E.E. Connor, M.J. Paape, J.L. Williams and D.D. Bannerman, 2005. Characterization of bovine FAS-associated death domain gene. Anim. Genet., 36: 63-66.

Tatischeff, I., F. Lavialle, S. Pigaglio-Deshayes, C. Pechoux-Longin, L. Chinsky and A. Alfsen, 2008. Dictyostelium extracellular vesicles containing hoechst 33342 transfer the dye into the nuclei of living cells: A fluorescence study. J. Fluorescence, 18: 319-328.

Yallop, C.A. and I. Svendsen, 2001. The effects of G418 on the growth and metabolism of recombinant mammalian cell lines. Cytotechnology, 35: 101-114.

Yamaguchi, M., S. Tomono and T. Nakagawa, 2007. Overexpression of RGPR-p117 suppresses apoptotic cell death and its related gene expression in cloned normal rat kidney proximal tubular epithelial NRK52E cells. Int. J. Mol. Med., 20: 565-571.

Yamamoto, T., E. Ueta and T. Osaki, 2003. Apoptosis induction by interleukin-2-activated cytotoxic lymphocytes in a squamous cell carcinoma cell line and Daudi cells-involvement of reactive oxygen species-dependent cytochrome $c$ and reactive oxygen species-independent apoptosis-inducing factors. Immunology, 110: 217-224.

Yu, Y., C. Iclozan, T. Yamazaki, X.X. Yang, C. Anasetti, C. Dong, and X.Z. Yu, 2009. Abundant c-Fas-associated death domain-like interleukin-1-converting enzyme inhibitory protein expression determines resistance of $\mathrm{T}$ helper 17 cells to activation-induced cell death. Blood, 114: 1026-1028.

Yu, Y.S., H.S. Sui, Z.B. Han, W. Li, M.J. Luo and J.H. Tan, 2004. Apoptosis in Granulosa cells during follicular atresia: Relationship with steroids and insulin-like growth factors. Cell Res., 14: 341-346. 